PROCEEDINGS OF THE

AMERICAN MATHEMATICAL SOCIETY

Volume 130, Number 7 , Pages 1997-2000

S 0002-9939(01)06270-0

Article electronically published on November 15, 2001

\title{
ON A CONJECTURED NONCOMMUTATIVE BEALS-CORDES-TYPE CHARACTERIZATION
}

\author{
SEVERINO T. MELO AND MARCELA I. MERKLEN \\ (Communicated by Christopher D. Sogge)
}

\begin{abstract}
Given a skew-symmetric matrix $J$, we prove that a bounded operator $A$ on $L^{2}\left(\mathbb{R}^{d}\right)$, for which $(z, \zeta) \mapsto T_{z} M_{\zeta} A M_{\zeta}^{-1} T_{z}^{-1}$ is smooth, and which commutes with all pseudodifferential operators $G(x+J D), G \in \mathcal{S}\left(\mathbb{R}^{d}\right)$, is of the form $F(x-J D)$, with $F$ possessing bounded derivatives of all orders on $\mathbb{R}^{d}$. Here, $T_{z}$ and $M_{\zeta}$ denote the translation and the gauge representations of $\mathbb{R}^{d}$. This was conjectured by Rieffel (1993) and is an application of the well-known Cordes' characterization of the the Heisenberg-smooth operators as pseudodifferential operators.
\end{abstract}

\section{IntRoduction}

Let $\mathbb{A}$ be a $\mathrm{C}^{\star}$-algebra with norm $\|\cdot\|_{A}$ and let $\mathcal{B}^{\mathbb{A}}\left(\mathbb{R}^{d}\right)$ denote the Fréchet algebra of all functions from $\mathbb{R}^{d}$ to $\mathbb{A}$ possessing bounded partial derivatives of all orders. Given a $d \times d$ real matrix $J$, a deformed product on $\mathcal{B}^{\mathbb{A}}\left(\mathbb{R}^{d}\right)$,

$$
F \times{ }_{J} G(x)=\iint F(x+J u) G(x+v) e^{2 \pi i u \cdot v} d u d v,
$$

was defined by Rieffel [6], with the above integral interpreted as an oscillatory one. If $J=0$, one then gets the pointwise product.

Let $\|\cdot\|$ denote the norm

$$
\|f\|=\left\|\int f(x)^{\star} f(x) d x\right\|_{A}^{\frac{1}{2}},
$$

defined for $f$ in the $A$-valued Schwartz space on $\mathbb{R}^{d}, \mathcal{S}^{\mathbb{A}}\left(\mathbb{R}^{d}\right)$, of all $f \in \mathcal{B}^{\mathbb{A}}\left(\mathbb{R}^{d}\right)$ such that sup $\left\|x^{\beta} \partial^{\alpha} f(x)\right\|_{A}$ is finite for all multi-indices $\alpha$ and $\beta$. If one assumes that $J$ is skew-symmetric, then the operator $g \mapsto L_{F}(g)=F \times_{J} g$ is bounded on $\mathcal{S}^{\mathbb{A}}\left(\mathbb{R}^{d}\right)$ with respect to the norm $\|\cdot\|$, for any $F \in \mathcal{B}^{\mathbb{A}}\left(\mathbb{R}^{d}\right)$ (cf. [6], Theorem 4.6). That result is a vector-valued version of Calderon-Vaillancourt theorem, since, if $G=g$ belongs to $\mathcal{S}^{\mathbb{A}}\left(\mathbb{R}^{d}\right)$, the oscillatory integral in (1) becomes an iterated convergent

Received by the editors November 29, 2000 and, in revised form, January 21, 2001.

2000 Mathematics Subject Classification. Primary 47G30.

Key words and phrases. Pseudodifferential operators, Heisenberg group.

The first author was supported in part by CNPq (Brazil), Processo 300330/88-0.

The second author was also supported by CNPq, Processo 142280/97-6. 
integral and we get

$$
L_{F} g(x)=\frac{1}{(2 \pi)^{d}} \int e^{i x \cdot \xi} F\left(x-\frac{J \xi}{2 \pi}\right) \widehat{g}(\xi) d \xi, \text { with } \widehat{g}(\xi)=\int e^{-i y \cdot \xi} g(y) d y .
$$

That is, $L_{F}$ is a pseudodifferential operator with $\mathbb{A}$-valued symbol

$$
a(x, \xi)=F\left(x-\frac{J \xi}{2 \pi}\right),
$$

which we denote by $L_{F}=a(x, D)=F\left(x-\frac{J D}{2 \pi}\right)$.

At the end of Chapter 4 of his monograph [6], Rieffel made a conjecture about $\left\{L_{F} ; F \in \mathcal{B}^{\mathbb{A}}\left(\mathbb{R}^{d}\right)\right\}$, which we rephrase as follows. That class should coincide with the set of all $\|\cdot\|$-bounded operators on $\mathcal{S}^{\mathbb{A}}\left(\mathbb{R}^{d}\right)$ (or on its $\|\cdot\|$-completion) which are smooth vectors for the usual action of the Heisenberg group and, in addition, commute with all operators $R_{G}(f)=f \times{ }_{J} G, G \in \mathcal{B}^{\mathbb{A}}\left(\mathbb{R}^{d}\right)$. Since $\times_{J}$ is associative, it is obvious that every $L_{F}$ commutes with the right multiplications. That they are smooth vectors for the action of the Heisenberg group follows, as in the scalar case (4], Section VIII.2), from the fact that the norm of $L_{F}$ is bounded by a continuous seminorm on $\mathcal{B}^{\mathbb{A}}\left(\mathbb{R}^{d}\right)$. So, every $L_{F}$ is as conjectured. We would like to point out in this paper that, already for the simplest case $\mathbb{A}=\mathbb{C}$, the converse to that statement is an interesting problem in pseudodifferential operators. Our result is stated below, in Theorem 1 which actually requires that the given bounded operator commutes only with $R_{G}$ for $G$ in $\mathcal{S}\left(\mathbb{R}^{d}\right)$.

Let $\mathcal{H}$ denote the Hilbert space $L^{2}\left(\mathbb{R}^{d}\right)$, and, for $z, \zeta \in \mathbb{R}^{d}$, and $f \in \mathcal{H}, T_{z} f(x)=$ $f(x-z)$ and $M_{\zeta} f(x)=e^{i \zeta \cdot x} f(x)$. Let $\mathcal{L}(\mathcal{H})$ denote the algebra of bounded operators on $\mathcal{H}$, with operator-norm also denoted by $\|\cdot\|$. Let us define an operator $A \in$ $\mathcal{L}(\mathcal{H})$ to be Heisenberg-smooth if the mapping $(z, \zeta) \mapsto T_{z} M_{\zeta} A M_{\zeta}^{-1} T_{z}^{-1}$ is (normtopology) infinitely differentiable. When $\mathbb{A}=\mathbb{C}$, we omit the superscripts and write $\mathcal{B}$ and $\mathcal{S}$ instead of $\mathcal{B}^{\mathbb{C}}$ and $\mathcal{S}^{\mathbb{C}}$.

Cordes proved [3] that an operator in $\mathcal{L}(\mathcal{H})$ is Heisenberg-smooth if and only if it is of the form $a(x, D)$, for some $a \in \mathcal{B}\left(\mathbb{R}^{2 d}\right)$. That is closely related to another abstract characterization of pseudodifferential operators proved by Beals [1]. We follow Taylor [8] in calling a result of that kind a Beals-Cordes-type characterization.

A generalization of the above-quoted Cordes result to the case of $\mathrm{C}^{\star}$-algebravalued symbols is the main obstacle for a full proof of Rieffel's conjecture. Once that is achieved, our proof of Theorem 1 could be adapted to the more general setting.

Theorem 1. Let $J$ be a skew-symmetric $d \times d$-matrix. If $A$ is a Heisenberg-smooth operator such that $A R_{G}=R_{G} A$, for all $G \in \mathcal{S}\left(\mathbb{R}^{d}\right)$, then $A=L_{F}$ for some $F \in$ $\mathcal{B}\left(\mathbb{R}^{d}\right)$.

In Lemma1, the symbols $a$ of the $L_{F}$ 's are described in terms of the symbols $b$ of the right $\times_{J}$-multiplications $R_{G}$ 's. It turns out that a Heisenberg-smooth operator is an $L_{F}$ if and only if all Poisson brackets $\{a, b\}$ 's vanish, with the corresponding $G$ 's ranging in a larger class, where the $R_{G}$ 's are not necessarily bounded.

In order to prove that every Heisenberg-smooth operator which commutes with the $R_{G}$ 's is the strong-operator limit of $L_{F}$ 's, we use the fact that every Heisenbergsmooth operator leaves $\mathcal{S}\left(\mathbb{R}^{d}\right)$ invariant (this follows from the above quoted Cordes' characterization of the Heisenberg-smooth operators as pseudodifferential operators), and Lemma 2 which means precisely that the norm-closure of $\left\{L_{F} ; F \in\right.$ $\left.\mathcal{S}\left(\mathbb{R}^{d}\right)\right\}$ is a nondegenerate $\mathrm{C}^{\star}$-subalgebra of $\mathcal{L}(\mathcal{H})$. Lemma 2 also implies that 
$\mathcal{S}\left(\mathbb{R}^{d}\right)$, with product $\times_{J}$, is a Hilbert algebra, in the sense of [5]. Hence, Theorem 1.11 of [7] implies that every bounded operator which commutes with the right regular representation belongs to the von Neumann algebra generated by the left regular representation. We stress, however, that this more general argument is not needed here, since the given operator is Heisenberg-smooth.

\section{Proof of Theorem 1}

Given smooth functions on $\mathbb{R}^{2 d}, a=a(x, \xi)$ and $b=b(x, \xi)$, let us define, as usual, their Poisson bracket by

$$
\{a, b\}=\sum_{j=1}^{d}\left(\frac{\partial a}{\partial x_{j}} \frac{\partial b}{\partial \xi_{j}}-\frac{\partial a}{\partial \xi_{j}} \frac{\partial b}{\partial x_{j}}\right) .
$$

Let us denote by $\mathcal{P}_{B}\left(\mathbb{R}^{d}\right)$ the space of all smooth complex functions on $\mathbb{R}^{d}$ which, together with all its derivatives, are polynomially bounded.

Lemma 1. Given a skew-symmetric $d \times d$-matrix $J$ and given $a \in C^{\infty}\left(\mathbb{R}^{2 d}\right)$, there exists $F \in C^{\infty}\left(\mathbb{R}^{d}\right)$ such that $a(x, \xi)=F(x-J \xi)$ if and only if $\{a, b\}=0$ for all $b$ of the form $b(x, \xi)=G(x+J \xi), G \in \mathcal{P}_{B}\left(\mathbb{R}^{d}\right)$.

Proof. Let $F$ and $G$ be any smooth functions on $\mathbb{R}^{d}$, and let $a(x, \xi)=F(x-J \xi)$ and $b(x, \xi)=G(x+J \xi)$. Then we have

$$
\{a, b\}=\sum_{k, l=1}^{d}\left(J_{l k} \partial_{k} F \partial_{l} G+J_{l k} \partial_{l} F \partial_{k} G\right),
$$

which vanishes, because $J_{k l}=-J_{l k}$.

For the converse, suppose that $a \in C^{\infty}\left(\mathbb{R}^{2 d}\right)$ is such that $\left\{a, b_{k}\right\}=0, k=$ $1, \cdots, d$, where $b_{k}(x, \xi)=G_{k}(x+J \xi), G_{k}\left(s_{1}, \cdots, s_{d}\right)=s_{k}$. Then, $a$ satisfies the system of linear partial differential equations

$$
\frac{\partial a}{\partial \xi_{k}}=\sum_{l=1}^{d} J_{k l} \frac{\partial a}{\partial x_{l}}, k=1, \cdots, d .
$$

This means that, for each $(x, \xi) \in \mathbb{R}^{2 d}$ and for each $k, a$ is constant along the line $\left\{\left(x-t\left(J_{k 1}, \cdots, J_{k d}\right), \xi_{1}, \cdots, \xi_{k}+t, \cdots, \xi_{d}\right) ; t \in \mathbb{R}\right\}$. Then, if we let $F(x)=a(x, 0)$, we get $a(x, \xi)=F(x-J \xi)$.

Lemma 2. Given a skew-symmetric $d \times d$-matrix $J$, there exists a sequence $E_{k} \in$ $\mathcal{S}\left(\mathbb{R}^{d}\right)$ such that $L_{E_{k}}$ converges to the identity in $\mathcal{L}(\mathcal{H})$, in strong-operator topology.

Proof. Let $\psi$ be a smooth, positive function, with support contained in the open unit ball centered at the origin, and with $\int \psi(\xi) d \xi=1$. For each non-negative integer $k$, let $\psi_{k}(\xi)=k^{d} \psi(k \xi)$, and let $\varphi_{k} \in \mathcal{S}\left(\mathbb{R}^{d}\right)$ be such that $\widehat{\varphi}_{k}=\psi_{k}$. It is elementary to prove that, if $F$ in $\mathcal{S}\left(\mathbb{R}^{d}\right)$, a non-negative integer $l$, and $\epsilon>0$ are given, then there exists $\delta>0$ such that

$$
|x|^{l}\left|F(x-J \xi / 2 \pi) e^{i x \cdot \xi}-F(x)\right|<\epsilon, \forall x \in \mathbb{R}^{d}, \text { if }|\xi|<\delta .
$$

If $k>\delta^{-1}$, then it follows from (2) that $|x|^{l}\left|(2 \pi)^{d} L_{F} \varphi_{k}(x)-F(x)\right|<\epsilon$, for all $x \in \mathbb{R}^{d}$. Thus, $s(x)=(1+|x|)^{d}$ is an example of a function such that $s^{-1} \in \mathcal{H}$ and $s L_{F}\left(\varphi_{k}\right)$ converges uniformly to $(2 \pi)^{-d} s F$. So, $F \times_{J} \varphi_{k}$ converges to $(2 \pi)^{-d} F$ in $\mathcal{H}$. 
It is easy to see that right $\times_{J}$-multiplication is the same as left $\times_{(-J)^{-}}$- multiplication. So, the argument of the previous paragraph applied with $(-J)$ replacing $J$ implies that, for all $F \in \mathcal{S}\left(\mathbb{R}^{d}\right), L_{\varphi_{k}}(F)$ converges to $(2 \pi)^{-d} F$ in $\mathcal{H}$. We will be finished, taking $E_{k}=(2 \pi)^{d} \varphi_{k}$, if we can show that sup $\left\|L_{E_{k}}\right\|$ is finite. This follows from $\varphi_{k}(x)=\varphi_{1}(x / k)$ and from the fact that $\left\|L_{F}\right\|$ is bounded by the sum of the supremums of a finite number of derivatives of $F$ ([2]; or [6], Chapter 4, for the $\mathbb{A}$-valued case).

Let $A$ satisfy the hypotheses of Theorem 1. Being Heisenberg-smooth, $A$ must be equal to $a(x, D)$ for some $a \in \mathcal{B}\left(\mathbb{R}^{2 d}\right)$. In particular, $A$ leaves $\mathcal{S}\left(\mathbb{R}^{d}\right)$ invariant. Define $F_{k}=A\left(E_{k}\right) \in \mathcal{S}\left(\mathbb{R}^{d}\right)$, with $E_{k}$ as in Lemma 2, Using that $A$ commutes with right $\times_{J}$-multiplications, and that $\mathcal{S}\left(\mathbb{R}^{d}\right)$ is dense in $\mathcal{H}$, we get $L_{F_{k}}=A L_{E_{k}}$. Hence, $A$ is the strong-operator limit of the sequence $L_{F_{k}}$.

Let $K_{k}$ and $K$ denote the Schwartz kernels of $L_{F_{k}}$ and $A$, respectively. The fact that $L_{F_{k}}$ converges to $A$ strongly implies that $K_{k}$ converges to $K$ with respect to the weak topology of $\mathcal{S}^{\prime}\left(\mathbb{R}^{2 d}\right)$. Using the well-known relation between the symbol and the Schwartz kernel of a pseudodifferential operator (see, for example, 44, Proposition I.2.6), we conclude that the symbols of the $L_{F_{k}}$ 's, $a_{k}(x, \xi)=F_{k}\left(x-\frac{J \xi}{2 \pi}\right)$, converge weakly in $\mathcal{S}^{\prime}\left(\mathbb{R}^{2 d}\right)$ to the symbol of $A, a(x, \xi)$. By Lemma $\left.1 a_{k}, b\right\}=0$ for every $b$ of the form $b(x, \xi)=G\left(x+\frac{J \xi}{2 \pi}\right), G \in \mathcal{P}_{B}\left(\mathbb{R}^{d}\right)$. Since $\{\cdot, b\}$ is a continuous operation on $\mathcal{S}^{\prime}\left(\mathbb{R}^{2 d}\right)$, we see that $\{a, b\}$ also vanishes for every such $b$. By the lemma again, $a(x, \xi)=F\left(x-\frac{J \xi}{2 \pi}\right)$ for some $F \in \mathcal{B}\left(\mathbb{R}^{d}\right)$, as we wanted to prove.

\section{ACKNOWLEDGEMENT}

We would like to thank Ruy Exel and Mark Rieffel for some friendly and helpful e-mail exchanges.

\section{REFERENCES}

[1] R. BEAls, Characterization of pseudodifferential operators and applications; Duke Math. J. 44, 45-57, 1977; ibid. 46, 215, 1979. MR 80b:47062

[2] A. P. Calderon \& R. Vaillancourt, On the boundedness of pseudodifferential operators, J. Math. Soc. Japan 23, 374-378, 1971. MR 44:2096

[3] H. O. Condes, On pseudodifferential operators and smoothness of special Lie-group representations, Manuscripta Math. 28, 51-69, 1979. MR 80m:47047

[4] H. O. Cordes, The technique of Pseudodifferential Operators, Cambridge Univ. Press, 1995. MR 96b:35001

[5] J. Dixmier, Von Neumann Algebras, North-Holland, 1981. MR 83a:46004

[6] M. Rieffel, Deformation Quantization for Actions of $\mathbb{R}^{d}$, Memoirs of the American Mathematical Society 506, 1993. MR 94d:46072

[7] M. Rieffel, Square-Integrable Representations of Hilbert Algebras, J. Funct. Anal. 3, 265300, 1969. MR 39:6094

[8] M. E. TAYLOR, Beals-Cordes-type characterizations of pseudodifferential operators, Proc. Amer. Math. Soc. 125-6, 1711-1716, 1997. MR 97g:35186

Instituto de Matemática e Estatística, Universidade de São Paulo, Caixa Postal 66281, 05315-970, SÃo PAUlo, BraZil

E-mail address: toscano@ime.usp.br

Instituto de Matemática e Estatística, Universidade de São Paulo, Caixa Postal 66281, 05315-970, São Paulo, Brazil

E-mail address: marcela@ime.usp.br 